\title{
SHORT-TERM DIETARY RESTRICTION IN OLD ZEBRAFISH CHANGES CELL SENESCENCE MECHANISMS
}

\author{
AYCA ARSLAN-ERGUL, , a,b,c BEGUN ERBABA, ${ }^{a, b, c}$ \\ ELIF TUGCE KAROGLU, ${ }^{a, b, c}$ DILARA OZGE HALIM ${ }^{d}$ AND \\ MICHELLE M. ADAMS ${ }^{a, b, c, e, f_{*}}$ \\ a Bilkent University, Interdisciplinary Neuroscience Program, \\ Ankara, Turkey \\ ${ }^{\mathrm{b}}$ Bilkent University, Molecular Biology and Genetics \\ Department Zebrafish Facility, Ankara, Turkey \\ ${ }^{\mathrm{c}}$ Bilkent University, UNAM-Institute of Materials Science and \\ Nanotechnology, Ankara, Turkey \\ ${ }^{\mathrm{d}}$ Bilkent University, Department of Molecular Biology and \\ Genetics, Ankara, Turkey \\ e Bilkent University, Department of Psychology, Ankara, Turkey \\ ${ }^{\mathrm{f}}$ Bilkent University, Aysel Sabuncu Brain Research Center, \\ Ankara, Turkey
}

\begin{abstract}
Brain aging is marked by a decline in cognitive abilities and associated with neurodegenerative disorders. Recent studies have shown, neurogenesis continues into adulthood but is known to be decreasing during advancing age and these changes may contribute to cognitive alterations. Advances, which aim to promote better aging are of paramount importance. Dietary restriction (DR) is the only non-genetic intervention that reliably extends lifeand health-span. Mechanisms of how and why DR and age affect neurogenesis are not well-understood, and have not been utilized much in the zebrafish, which has become a popular model to study brain aging and neurodegenerative disease due to widely available genetic tools. In this study we used young (8-8.5 months) and old (26-32.5 months) zebrafish as the model to investigate the effects of a shortterm DR on actively proliferating cells. We successfully applied a 10-week DR to young and old fish, which resulted in a significant loss of body weight in both groups with no effect on normal age-related changes in body growth. We found that age decreased cell proliferation and increased senescence associated $\beta$-galactosidase, as well as shortened telomere lengths. In contrast, DR shortened telomere lengths only in young animals. Neither age nor DR changed the differentiation patterns of glial cells. Our results suggest that the potential effects of DR could be mediated by
\end{abstract}

\footnotetext{
${ }^{*}$ Correspondence to: M. M. Adams, Interdisciplinary Neuroscience Program, Bilkent University, Aysel Sabuncu Brain Research Center, 06800 Bilkent, Ankara, Turkey. Fax: +90-312-290-2561.

E-mail addresses: aycaergul@bilkent.edu.tr (A. Arslan-Ergul), begun. erbaba@bilkent.edu.tr (B. Erbaba), elif.karoglu@bilkent.edu.tr (E. T. Karoglu), do.halim@gmail.com (D. O. Halim), michelle@bilkent.edu. $\operatorname{tr}$ (M. M. Adams).

Abbreviations: BrdU, bromodeoxyuridine; DR, dietary restriction; gfap, glial fibrillary acidic protein; GFP, green fluorescent protein; qPCR, quantitative polymerase chain reaction; SA- $\beta$-gal, senescence associated $\beta$-galactosidase; TRF, telomeric terminal restriction fragment.
}

telomere regulation and whether these are beneficial or negative remains to be determined. (c) 2016 IBRO. Published by Elsevier Ltd. All rights reserved.

Key words: aging, zebrafish, brain, dietary restriction, cell proliferation, telomere.

\section{INTRODUCTION}

While there has been progress made toward understanding the complexity of brain aging, our comprehension is still limited. In order to determine the appropriate interventions to alter the course of brain aging and age-associated neurological disorders, we must first understand the normal age-related changes that occur. Previously it was thought that brain cells were not replenishing but research that has been performed over the last decade has indicated otherwise. For example, a recent study revealed that approximately 700 new neurons are added daily to each hippocampus (Spalding et al., 2013). Moreover, there is evidence that the progenitor cell proliferation is reduced in old brains (Kempermann et al., 1998; Luo et al., 2006). Therefore, it is evident that there are stem cells residing in young and old brains and they constantly but slowly give rise to neurons and glia. However, even though it is the case that new neurons and glia are produced, not all cells make it to maturation.

It is clear that external factors can affect neurogenesis. For example, learning enhances neurogenesis in the hippocampus (Gould et al., 1999), and exercise, specifically running, helps make functional hippocampal neurons (van Praag et al., 1999; van Praag, 2009). The oligodendrocyte precursor cell proliferation doubles during sleep and positively correlates with time spent in REM sleep, whereas the differentiation is higher during wakefulness (Bellesi et al., 2013). Thus, although cell proliferation occurs in the aging brain, it is reduced compared to young and is very likely affected by external factors.

Dietary restriction (DR) is the only non-genetic intervention that reliably increases life-span. Numerous studies have demonstrated that a caloric reduction in food intake from ad libitum levels, i.e. DR, increases life-span (Roth et al., 2001; Lin et al., 2002; Colman et al., 2009). DR also has been shown to affect neurogenesis, as well as neural progenitor cells. For example, DR enhances the survival of newly born neurons 
(Park and Lee, 2011). Long-term DR leads to an increase in proliferation of hippocampal stem cells in female mice (Park et al., 2013). Moreover, DR increases both bromodeoxyuridine (BrdU) positive cells (Kitamura et al., 2006), and the survival rates of the cells in the dentate gyrus of mice (Lee et al., 2002). Thus, DR alters cell proliferation in an area of the brain that is sensitive to age-related decline, and may change the course of age-related cognitive decline.

The zebrafish has become a popular model organism to study the effects of brain aging and age-associated disorders. It has a similar genome to humans and ages gradually; living on average between 3 and 5 years (Kishi et al., 2003; Howe et al., 2013). These animals have an integrated nervous system and exhibit advanced behavioral properties like memory and social behavior (Kishi et al., 2009). The zebrafish brain has regenerative properties and neurogenesis. However, in contrast to mammals, neurogenesis is not restricted to the telencephalon but is widespread throughout the entire brain (Kizil et al., 2012; Schmidt et al., 2013). Proliferating cells are frequent on the outer edges of the zebrafish telencephalon. Decreases in neurogenesis during aging have also been shown in zebrafish and were correlated with the silencing of radial glia (Edelmann et al., 2013). Thus, the zebrafish animal model has equivalent properties to humans and is a good model for understanding human brain aging, especially in the context of changes in neurogenesis.

The purpose of this study was to examine the effects of short-term DR on the early phase of cell proliferation in young (8-8.5 months) and old (26-32.5 months) animals. For this, we labeled the cells with BrdU for $4 \mathrm{~h}$, enabling the detection of actively proliferating cells. We were interested in the effects of DR on cell proliferation across the whole telencephalon, as well as possible regional differences because previous studies in the non-human primate and rodent hippocampus have indicated that areas of the hippocampus are differentially affected by aging and diet (Gazzaley et al., 1996; Rapp and Gallagher, 1996; Smith et al., 2000; Adams et al., 2001, 2010). Our aim was to identify the patterns of cell proliferation that change between young and old animals with and without DR. We included in our studies a transgenic zebrafish line [glial fibrillary acidic protein (gfap): green fluorescent protein (GFP)], which enabled us to distinguish proliferating cells of the glial origin. In addition to these studies we also examined the markers associated with cellular senescence, such as telomere lengths and senescence associated $\beta$-galactosidase (SA- $\beta$-gal) activity in the brain. To our knowledge, this is one of the first studies to analyze short-term DR and cell proliferation in old subjects, as well as the first to analyze DR and cell proliferation in young and old zebrafish.

\section{EXPERIMENTAL PROCEDURES}

\section{Animals}

Overall, 118 adult zebrafish were used in this study. All zebrafish were kept in standard systems built by ZebTec Zebrafish Housing System (Tecniplast, Italy), at $28^{\circ} \mathrm{C}$ water, with $14-\mathrm{h}$ light: 10 -h dark cycles. Fish were fed every day, two times a day with dry flakes and three times a week with fresh artemia. All the fish used in the experiments in this study were adult fish; older than 8 months old; young fish were 8-8.5 months old, and old fish were 26-32.5 months old. Fish were either of wild-type $A B$ strain, which was raised and bred in our fish facility, or TgBAC(gfap-GFP)zf167, which was obtained from the European Zebrafish Resource Center. The gfap:GFP line was used only in triple staining experiments in order to characterize the origin of the proliferating cells. Ten-liter tanks were used for the experimental set-up and densities were kept between 8 and 15 fish. The dates of birth were recorded and only fish with the same birth dates were kept together in the same tank. To confirm the gender of the fish, gonad examination was done by directly observing testes or eggs. Fish without any observable gonad were registered as "no gonad". We included both males and females in all our cohorts in order to represent both genders in our studies.

For the feeding experiments, fish were divided into two groups, ad libitum (AL) or dietary restricted (DR). Firstly, all the experimental fish of the same age were combined into a single tank, and then divided into two tanks, randomly. Fish were weighed prior to and after the experiment. AL fish were fed a spoonful of dry flakes using a Starbucks green drink plug, which is approximately $90 \mathrm{mg}$, twice a day, and also with artemia, a small shrimp, three times a week. DR fish received a spoonful of dry flakes once in two days, and artemia once a week. The diet continued for 10 weeks and the two groups of fish were kept on the same shelf, adjacent to each other.

Experiments were conducted in separate cohorts; for each set, fish with the same date of birth were pooled together, and then were assigned randomly to the DR and $\mathrm{AL}$ groups. Fish were allowed to habituate to their new tank for at least three days, and then the feeding schedules began. After the fish were habituated and the feeding scheme had started, the fish remained in the same tank throughout the entire experiment to prevent disturbances in the social network. At the start and end of the experiments, the fish were weighed in a beaker full of water, either individually (old fish), or in groups, which is labeled as wet-weight. After the fish were euthanized, they were weighed on a piece of parafilm (dry weight) and the body lengths were measured. Body length was measured from the mouth to the end of the tail, which was the very anterior to the posterior end of the fish.

The animal protocol for this study was approved by the Bilkent University Local Animal Ethics Committee (HADYEK) with the approval date: Sept 3, 2012, no: 2012/24 and with the approval date: Jan 8, 2012, no: $2013 / 2$.

\section{Cortisol measurements}

Cortisol extraction from zebrafish was performed according to the protocol described in a previous study (Pavlidis et al., 2011). For the cortisol measurements, 
the Cortisol EIA Kit (ACE, 500360) was used and performed according to manufacturer's instructions. Fish were anesthetized in ice-water $\left(4^{\circ} \mathrm{C}\right)$ and after $10 \mathrm{~min}$, when all the movement ceased, the fish were euthanized by decapitation. Fish trunks, not the heads, were used as samples. Trunks were snap frozen in liquid nitrogen immediately after euthanization and stored at $-80^{\circ} \mathrm{C}$. On the day of the testing procedure, frozen trunks were immersed in cold PBS, weighed, homogenized, mixed with diethyl ether, separated into the phases and cortisol was recovered after overnight evaporation. Samples were diluted to $1 / 4$ and $1 / 8$ and tested in duplicates.

\section{BrdU injections}

For BrdU injections, the fish were transferred from the main tanks to the $17^{\circ} \mathrm{C}$ system water, which was then gradually dropped to $12^{\circ} \mathrm{C}$ by adding ice cubes. When the movement ceased, $10-20 \mu \mathrm{L}$ of a BrdU (Sigma, St Louis, MO, USA, B5002) solution, with a concentration of $10 \mathrm{mg} / \mathrm{mL}$ in water, was injected intraperitoneally into the fish. Immediately, the fish were transferred to aquaria with fresh system water at $28^{\circ} \mathrm{C}$. Recovery was closely observed, fish were fed with flakes and eating behavior and movements were monitored. After $4 \mathrm{~h}$, fish were transferred to ice-water to be euthanized.

\section{Immunostaining}

A detailed protocol can be found on Protocol Exchange (Arslan Ergul et al., 2013). Briefly, the head was immersed in fixation buffer (FB001, Invitrogen, Carlsbad, $\mathrm{CA}, \mathrm{USA})$ overnight in the refrigerator $\left(4^{\circ} \mathrm{C}\right)$, washed with PBS, then the brain was dissected by removing the skull, gills, and extra fat tissue. Following dehydration and rehydration steps in methanol, the whole brain was embedded in $3 \%$ agarose in PBS and after solidifying, was cut into slices of $50 \mu \mathrm{m}$ thickness with a Leica Vibratome VT1200S. Slices were washed with PBS-T, treated with $\mathrm{HCl}$, borax buffer, and blocked with PBS-T containing normal goat serum (Sigma). Primary antibody incubation at $4{ }^{\circ} \mathrm{C}$ overnight was followed by secondary antibody incubations at room temperature for two hours. Slices were mounted with Prolong antifade reagent (P36934, Invitrogen). The antibodies used were anti-BrdU antibody, mouse monoclonal (\#5292S, Cell Signaling Technologies, Danvers, MA, USA), anti-GFP antibody (ab13970, Abcam, Cambridge, UK), RabMab anti-NeuN antibody (ab177487, Abcam), anti-HuC antibody (ab78467, Abcam), anti-chicken Alexa Fluor 647 (ab150175, Abcam), anti-mouse Alexa Fluor - 488 secondary antibody raised in goat (\#4408, Cell Signaling Technologies), and anti-rabbit Alexa Fluor - 555 (\#4413, Cell Signaling Technologies). The nuclear stain used was a 7-AAD nuclearstain (A1310, Invitrogen). Images were taken with the Zeiss LSM 510 confocal microscope. For each sample, 6-8 sections were scanned with 10-15 layers at the $z$-axis, and 4-6 squares at the $x-y$ axis. Composite images were created with Image J (NIH, Bethesda, MD, USA).

\section{Telomere length measurements}

In order to measure telomere lengths, the quantitative polymerase chain reaction (qPCR) method was utilized. DNA from the brain, tail, spleen, and muscle samples was isolated with a QIAamp DNA Mini Kit (Qiagen, Redwood City, CA, USA, 51304). The telomere primers were adapted from a previous study (O'Callaghan and Fenech, 2011). As a reference, any single copy gene can be used, so here deiodinase, iodothyronine, type II (dio) was utilized. $\Delta \mathrm{Ct}$ was calculated as $\mathrm{Ct}$ (telomere) $\mathrm{Ct}$ (dio). Basically, the hypothesis is that the telomere primers will bind more whenever the telomeres are longer and they can be quantified using the GPCR method.

In order to strengthen the findings from the GPCR analyses about the DR effect on telomere lengths a TeloTAGGG Telomere length assay kit (Roche, Istanbul, Turkey) was used for the telomeric terminal restriction fragment (TRF) assay and the manufacturer's instructions were strictly followed. Only brain samples were included in the TRF assay and there were three samples per group. These samples were a subset of the same samples used in the qPCR analyses. Analysis of the gel image was performed using Telomeric software (Fox Case Cancer Center, USA). The calculations were performed in the same manner as described in a previous study (Terai et al., 2013).

\section{Senescence associated beta galactosidase assay (SA-p-gal)}

To measure senescence $\beta$-galactosidase, the SA- $\beta$-gal staining kit (9860S, Cell Signaling Technologies) was used according to the manufacturer's instructions. For the samples tested, similar steps were used as those described in the immunostaining protocol prior to the methanol procedures. In order to quantify the images, we utilized ImageJ's region of interest measurements. Blue areas were matched with threshold markings, and the signal was measured as a percentage of the selected area. Quantification was done using an unbiased and a blind analysis; i.e., the people who quantified the data were unaware of the sample IDs and are not authors on this paper.

\section{Statistical analysis}

All data were tested for a normal distribution and homogeneity of variance using the Kolmogorov-Smirnov and Levene's tests with the significance level set as 0.05. In the cases where these assumptions were violated, either a Kruskal-Wallis, a Wilcoxon signed rank or a Friedman test was applied. To determine differences between two groups, a Mann Whitney $U$ test was applied post hoc with a corrected $p$ value according to the number of comparisons between the groups. When the assumptions of a normal distribution and homogeneity of variance were not violated, a two-way analysis of variance (ANOVA) with the factors of diet and age was applied to the data sets. Following the ANOVA analysis, a post hoc comparison with a Bonferroni correction was used to determine between 
which groups a significant difference occurred. For all these analyses the statistical program SPSS (IBM, Istanbul, Turkey) was used.

\section{RESULTS}

\section{Weight loss occurs with dietary restriction without an evident increase in stress}

A modified version of every-other-day feeding for 10 weeks was the DR regimen used in the current study for 10 weeks. Other than the feeding schedule, all the fish were treated in the exact same manner. We observed that the food was in excess for ad libitum $(A L)$-fed fish, with uneaten food always observed at the bottom of the tank. Fig. 1 shows the average final body weight and length for each group. Since the initial measures were the same within the young and old groups only final measures are given. The wet weight measurements are available if requested.

At the end of the 10-week DR, restricted animals weighed significantly less than those fed $A L$. Our data demonstrated that $A L-f e d$ young animals weighed on average $0.57 \mathrm{~g}( \pm 0.04, n=34)$, whereas DR young animals had an average weight of $0.43 \mathrm{~g}( \pm 0.03$, $n=28)$. Old animals weighed $0.79 \mathrm{~g}( \pm 0.035, n=29)$, when fed with the $\mathrm{AL}$ diet, and $0.64 \mathrm{~g}( \pm 0.036, n=27)$ with a DR diet. Thus, young DR animals have a $25 \%$ weight reduction as compared to $A L$ young, and old $D R$ animals weighed $19 \%$ less than old $A L$ animals. This weight loss was significant within the age and diet groups, as demonstrated by a main effect of age and diet using a Kruskal-Wallis test [Fig. 1A; $H(3)=45$, $p<0.0005$, A Bonferroni correction was applied and so all effects are reported at a 0.008 level of significance. Significant differences, as measured by post hoc comparisons using a Mann Whitney $U$ test, were observed between the $C R-Y$ and $A L-Y$ groups $(U=191, r=-0.42), C R-Y$ and CR-O groups $(U=$ 135, $r=-0.55)$, CR-Y and AL-O groups $(U=66.50$, $r=-0.72), \quad A L-Y$ and CR-O groups $(U=252, r=$ $-0.38), A L-Y$ and AL-O groups $(U=176.50, r=-0.55)$ and CR-O and AL-O groups $(U=224.50, r=-0.37)]$. Thus our data indicated as expected that old animals weighed significantly more than the young animals, which is similar to the aging profile of rodents (Shi et al., 2007; Adams et al., 2008; Newton et al., 2008; Fig. 1A). Moreover, the 10-week every-other-day feeding significantly reduced the body weight as compared to the AL-fed groups.

The average body lengths increased in the aged animals as compared to young animals. On average the lengths were $3.7 \mathrm{~cm}( \pm 0.105, n=19)$ for $A L-Y, 3.5 \mathrm{~cm}$ $( \pm 0.08, n=25)$ for DR-Y, $4.2 \mathrm{~cm}( \pm 0.05, n=26)$ for $\mathrm{AL}-\mathrm{O}$, and $4.08 \mathrm{~cm}( \pm 0.08, n=24)$ for DR-O. Statistical analysis of our data indicated that with age body length increased significantly $[H(3)=40.07$, $p<0.0005$. A Bonferroni correction was applied and so all effects are reported at a .008 level of significance. Significant differences were observed between CR-Y and CR-O groups $(U=77.50, r=-0.64), C R-Y$ and AL-O groups $(U=41, r=-0.76), \quad A L-Y$ and $C R-O$
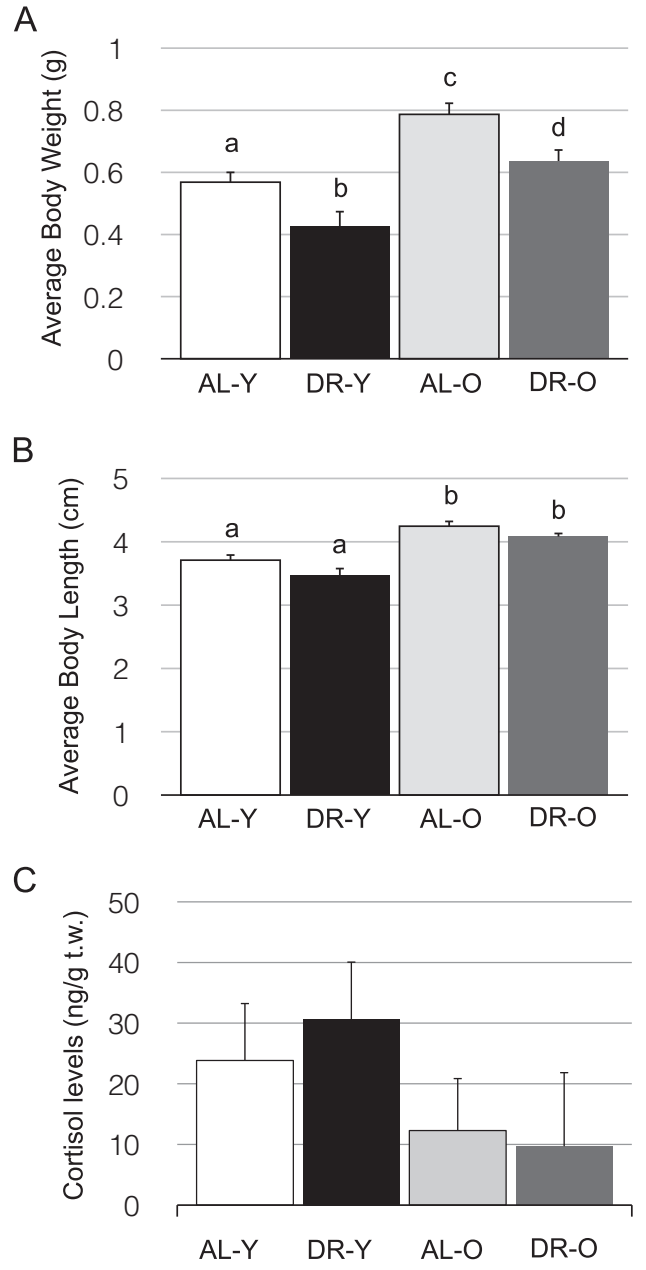

Fig. 1. An every-other-day feeding DR regimen significantly decreases body weight but does not alter body length or cortisol levels. (A) Average final body weight is reported in grams (In the AL-Y group $(n=34)$, in the DR-Y group $(n=28)$, in the AL-O group $(n=29)$ and in the DR-O group $(n=27)$. (B) Average final body length is reported in $\mathrm{cm}$. (C) Average trunk cortisol levels were measured from the following zebrafish groups: AL-Y $(n=5)$, DR-Y $(n=5)$, AL-O $(n=6)$, and DR-O $(n=3)$. Values on the $y$-axis are the average cortisol concentrations divided by the trunk weight (t.w.) and reported in nanograms/gram of trunk weight. AL, ad libitum; DR, dietary restricted; Y, Young (8-8.5 months) and O, Old (2632.5 months). Error bars represent the $+\mathrm{SE}$. Means with no common superscript letters are significantly different.

groups $(U=111.50, r=-0.44)$ and $A L-Y$ and $A L-O$ groups $(U=83.50, \quad r=-0.57)]$. Thus, the dietary regimen that we used did not have any effect on the body length of the animals [Fig. 1B; No significant difference was observed between $C R-Y$ and $A L-Y$ groups $(U=159, r=-0.29)$ and $C R-O$ and AL-O groups $(U=230.50, r=-0.23)]$. Therefore, it likely did not interfere with normal growth patterns.

Next we wanted to eliminate stress as a confounding factor and for this purpose, we performed an experiment in which we utilized a kit to measure the body cortisol levels of the fish. We employed a cortisol measurement protocol that gives reliable and consistent results (Pavlidis et al., 2011; Fig. 1C). We examined whether there were significant differences among the AL-fed and 
DR young and old groups. The analysis revealed that there are no significant differences between the groups (Fig. 1C; $H(3)=2.11, p=0.55$ ). Our data demonstrated no significant difference between the AL and DR groups $(U=34, z=-0.83, p=0.41, r=-0.19)$, nor the young and old groups $(U=29, \quad z=-1.31, \quad p=0.19$, $r=-0.30)$. Hence, diet does not appear to induce any additional stress to the fish in our experimental groups. Therefore, our data indicate that a 10-week DR successfully results in weight loss without interfering with normal age-related increases in body length or altering stress levels in the young and old fish.

\section{Dietary restriction does not alter the number of actively proliferating cells per unit volume}

We investigated the number of actively proliferating cells in the telencephalic region of the zebrafish, i.e. the cells that are proliferating in the narrow window of $4 \mathrm{~h}$. We started sectioning posterior to the olfactory bulb, and stopped anteriorly to the diencephalon. All sections were collected into plates and stained for BrdU. Images were captured using a confocal microscope and layers in the $z$-axis and tiles in the $x-y$ axis were combined into composite images (Fig. 2A). We calculated the number of cells per $\mathrm{mm}^{3}$ (cells $/ \mathrm{mm}^{3}$ ) in order to have a direct comparison between the sample groups that would control for changes in brain size between young and old animals. The cells $/ \mathrm{mm}^{3}$ calculation normalized the proliferation rates to the surface area and the depth of the sample that was scanned in order to prevent the probable bias of counting more cells when the brain volume gets larger. Cell counts can be normalized using only depth, however, since the older brains have a larger surface area, we included the area into the calculation as well.

We observed that the cells $/ \mathrm{mm}^{3}$ calculation decreases significantly with old age (Fig. $2 \mathrm{~B}, \mathrm{C} ; U=68, z=-2.58$, $p=0.01, r=-0.44)$, but not with diet $(U=137$, $z=-0.24, p=0.81, r=-0.04)$. The AL-Y group had an average of $3571 \mathrm{cells} / \mathrm{mm}^{3}(n=8, \pm 581)$, whereas DR-Y had 3240 cells $/ \mathrm{mm}^{3}(n=7, \pm 410)$, and the AL-O group had 2444 cells $/ \mathrm{mm}^{3}(n=10, \pm 421)$ and DR-O had $2073 \mathrm{cells} / \mathrm{mm}^{3} \quad(n=9, \quad \pm 395)$. Significant differences in cell numbers were not seen between the AL-Y, DR-Y, AL-O and DR-O groups $(H(3)=6.83$, $p=0.078$. Hence diet does not affect the cells $/ \mathrm{mm}^{3}$ ratios significantly. Moreover, global cell proliferation rates might not be differing significantly by diet but there may be regional differences within the telencephalon, and so this analysis was subsequently performed.

\section{Proliferation rates differ across telencephalic regions but not affected by age or diet}

We divided the brain slices into different telencephalic regions using a simplified version of the zebrafish brain atlas by Wullimann (Wullimann et al., 1996) and reduced the z-dimension, which resulted in 6 distinct regions (Fig. 3). Regions 1, 2, 3, and 6 have symmetrical counterparts; $1^{\prime}, 2^{\prime}, 3^{\prime}$, and $6^{\prime}$. We denoted them as such because imperfections in slicing could induce some discrepancies although the brain is symmetrical and the number of proliferating cells should be equal. Therefore, we counted the cells in both areas in each hemisphere. We designated the area in between the hemispheres as one region, and only divided this zone into two parts; 4 and 5, without symmetrical counterparts. Results demonstrated consistent proliferation rates between symmetrical areas; areas 1 and $1^{\prime}(T=238,50, p=0.45, r=-0.09), 2$ and $2^{\prime}$ $(T=250, p=0.42, r=-0.10), 3$ and $3^{\prime}(T=193$, $p=0.60, r=-0.06)$ and 6 and $6^{\prime}(T=205, p=0.27$, $r=-0.13$ ) had highly similar proliferation patterns, and only slight numerical variations between experimental groups. Areas 3 and $3^{\prime}$ had the lowest rates of proliferation overall. Strikingly, areas 4 and 5 had the highest rates of cell proliferation and also had the highest variation among the groups. According to our data, cell proliferation rates numerically decreased in old samples as compared to young samples but seemed to be unaffected by the diet (Fig. 3). However differences were not found to be statistically significant in either the different age or diet groups. Interestingly, although there were no effects of age or diet, the cell proliferation rate was significantly different among the different regions of the telencephalon as measured with a Friedman test $\left(\chi^{2} 2(9)=177.73, p<0.0005\right)$.

\section{Telomere length is shortened by DR in brain}

In a separate cohort of animals we determined whether DR affects the telomere lengths, which is a well-known biological counter that shortens with every cell division. The telomere length measurement protocol was adapted from a previous study (O'Callaghan and Fenech, 2011). The telomere lengths were measured in old and young zebrafish, in both the AL-fed and DR groups. We measured telomere lengths in selected zebrafish organs to observe the effect of diet and age on the telomeres. We tested the brain, muscle, spleen, and tail tissues (Fig. 4A-D, respectively). Although each tissue differs from the other significantly $(H(3)=18.99$, $p<0.0005)$; the brain and muscle tissues had relatively similar telomere lengths, and the tail and spleen had two and three fold longer telomeres, respectively. The telomere length variation is an interesting finding and it is reasonable considering the regenerative rates of the spleen and tail.

Two major outcomes were observed in the results. In the brain, using a two-way ANOVA, a significant main effect of treatment on telomere lengths was found $(F(1,15)=5.28, \quad p=0.039)$, but there was no significant main effect of age $(F(1,15)=1.5$, $p=0.240)$. For the telomere length in the spleen, on the other hand, we observed a significant main effect of age $(F(1,15)=8.65, p=0.010)$, but no significant main effect of the diet $(F(1,15)=0.80 p=0.384)$. We did not find any significant effects of age and diet on the telomere lengths in the tail $(H(3)=0.37, p=0.95)$ and muscle $(H(3)=5.11, p=0.16)$. Hence, although there are no significant effects of DR on cell proliferation rates, diet could have a negative impact on telomere lengths in the brains by shortening them (Fig. 4).

In order to substantiate the results in the brain telomere lengths, we have performed a TRF assay 
A

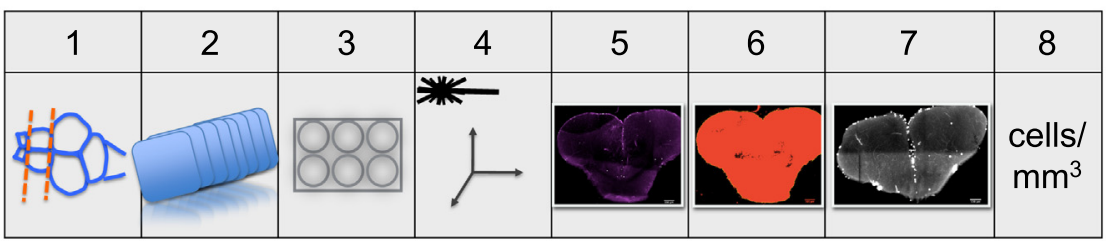

B

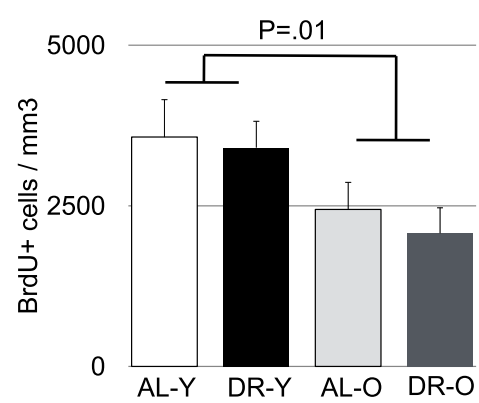

C

AL
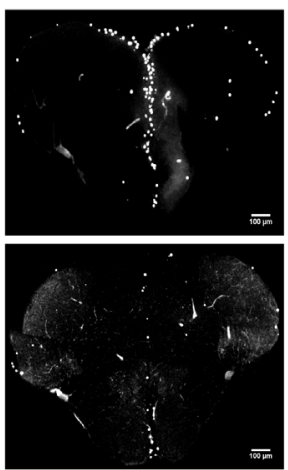

DR
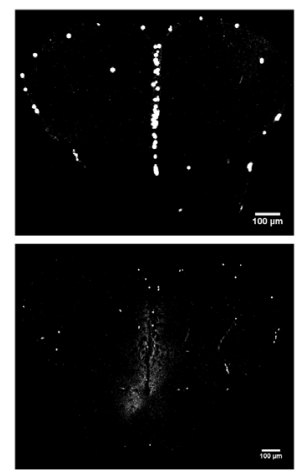

Fig. 2. Age but not diet affects cell proliferation rates in the telencephalon. (A) A description of the methods for detecting actively proliferating cells in telencephalon, i.e. overview of the experimental protocol. (1) A schematic drawing of a rostral view of zebrafish brain, where the cells were imaged and counted, telencephalon is indicated with dashed orange lines, (2) $50 \mu \mathrm{m}$ thick slices were obtained using a vibratome, (3) slices were taken into plates for staining, (4) slices were scanned with the confocal microscope, (5) composite images were created by Image J, (6) depth and area were measured for each slice, (7) proliferating cells were counted, and (8) proliferation is calculated per volume. (B) Cell proliferation rates for groups AL-Y $(n=8)$, DR-Y $(n=7)$, AL-O $(n=10)$, and DR-O $(n=9)$. The values are given as [the number of BrdU positive cells/volume in mm ${ }^{3}$ ]. C) Representative images from each group. BrdU positive cells are in white. Scale bars $=100 \mu \mathrm{m}$. AL, ad libitum; DR, dietary restricted; Y, Young (8-8.5 months) and O, Old (26-32.5 months). Error bars represent the + SE. (For interpretation of the references to colour in this figure legend, the reader is referred to the web version of this article.)

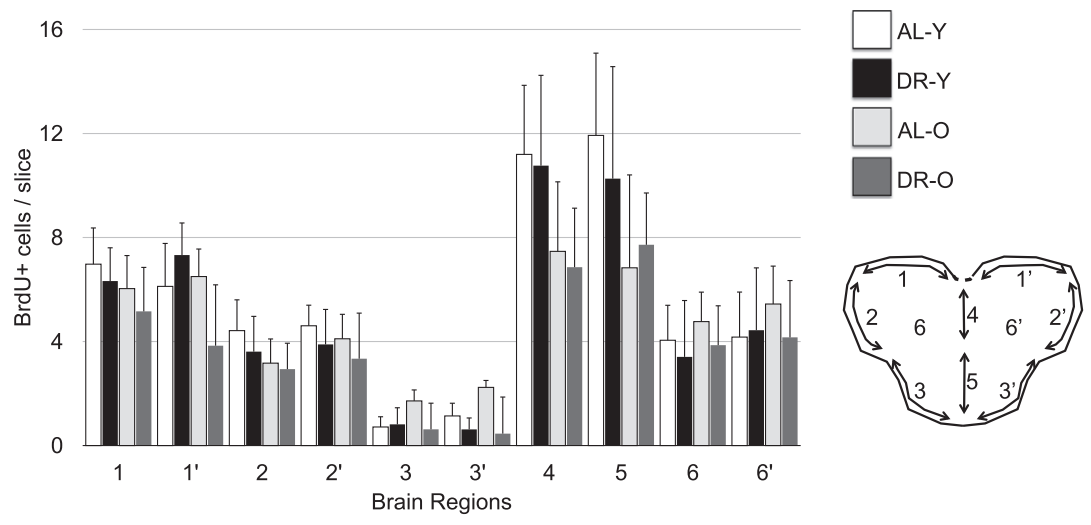

Fig. 3. Age and diet do not affect the number of actively proliferating cells in a region-specific manner. Distribution of actively proliferating cells in the various regions of the telencephalon. Corresponding regions are represented and shown on the diagram in the lower right hand corner. AL-Y $(n=8)$, DR-Y $(n=7)$, AL-O $(n=10)$, and DR-O $(n=9)$. AL, ad libitum; DR, dietary restricted; Y, Young (8-8.5 months) and O, Old $(26-$ 32.5 months). Error bars represent the $+\mathrm{SE}$.

(Fig. 5). The assay we chose was a non-radioactive version of the most common technique to measure telomere lengths. This assay involves usage of a kit that has been routinely utilized for measuring telomere lengths from human and rodent samples. We employed the same kit for the zebrafish brain samples. We repeated the test twice and quantified the bands from multiple exposures with the Telomeric software. One important thing to consider here is that we quantified the bands exactly as it is described in a previous study (Terai et al., 2013). Accordingly, the measurement rectangles were drawn from immediately above and below the highest and lowest MW bands, respectively. However, as opposed to human samples, in Terai et al., and lane 13 (Fig. 5A), there were smears above the rectangles. We tried to include all the bands, starting with the 
A
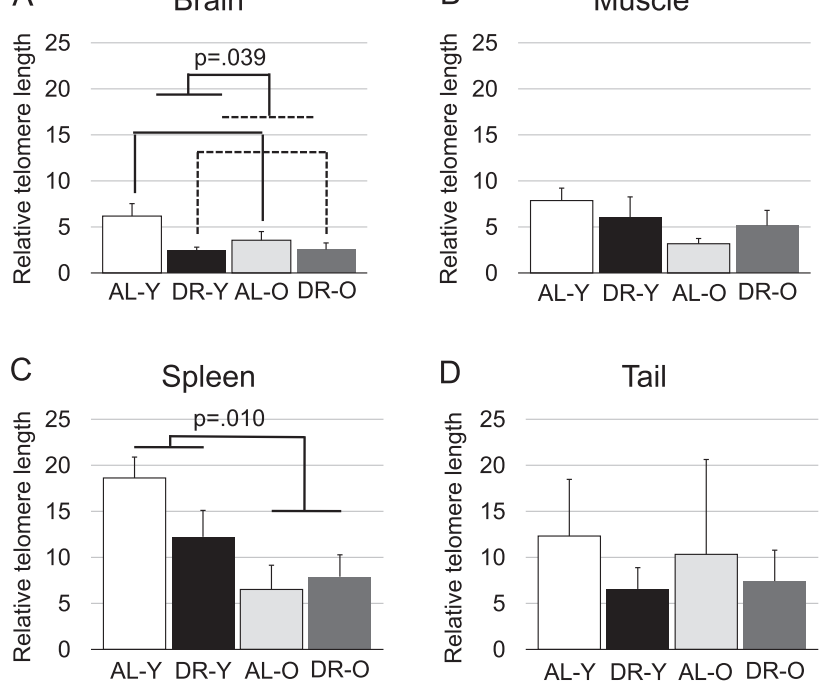

Fig. 4. Age and diet differentially affect telomere lengths in various zebrafish organs. Relative telomere lengths for zebrafish organs were measured by qPCR from genomic DNA. Telomere lengths were calculated as a relative value for (A) Brain, (B) Muscle, (C) Spleen, and (D) Tail. Telomere expression was normalized to a single copy gene (Dio) expression (For AL-Y $(n=6), \mathrm{DR}-\mathrm{Y}(n=6), \mathrm{AL}-\mathrm{O}$ $(n=3)$, and DR-O $(n=4))$. AL, ad libitum; DR, dietary restricted; $\mathrm{Y}$, Young (8-8.5 months) and O, Old (26-32.5 months). Error bars represent the +SE.

rectangles from the wells, but this caused all the TRF values to increase to a measurement above $30 \mathrm{kbps}$, which is not expected. Also the positive control sample was reported to be around $10 \mathrm{kbps}$, which is verified with the measurement technique used here.

After we quantified the bands from the southern blot gel image, the TRF median values were plotted as average values with the standard error of the groups. Although the findings in this experiment were not significant, $F(3,12)=1.022, p=0.443$ (Fig. 5B), most likely due to a small sample size, (3 per group in this figure, 6 per DR-Y and AL-Y samples due to the high concentration of DNA required for TRF measurements), numerically there was a trend for the TRF assay results to be in accordance with the data from the GPCR experiments demonstrating that in the young group the telomere lengths were shortened with DR (Fig. 4A). The QPCR method we used for the experimental results reported in Fig. 4 is more sensitive since it requires minimal amounts of DNA, and more accurate in terms of quantification. We measured the accuracy of the TRF assay by using the same zebrafish tissue samples included in the GPCR experiments. Thus, while there were no significant effects observed in this assay, we did see numerical trends toward a similar pattern and, therefore, are more confident in the GPCR results.

\section{Cellular senescence is affected by age but not by diet}

Another measure of cellular aging is the SA- $\beta$-gal assay, which is a highly common marker of senescence. The SA- $\beta$-gal assay measures the amount of betagalactosidase deposited in a cell, which increases in
A
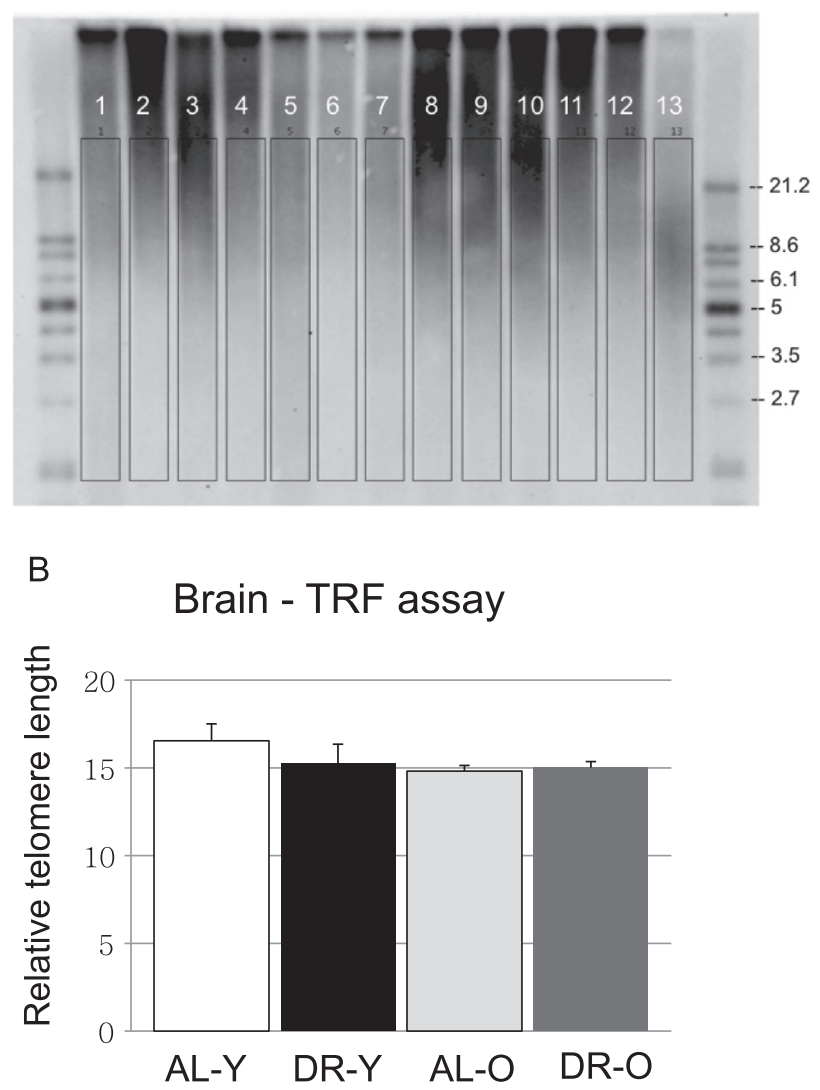

Fig. 5. The TRF assay for the zebrafish brain samples demonstrated a similar pattern of results to the GPCR experiments although not statistically significant. (A) The gel image is the Southern blot image from the TRF assay. The TeloTAGGG telomere length assay (Roche) was used. Lane numbers are marked with white numbers in the top portion of the gel. The first and last lanes include marker; the weights of the bands are displayed on the right hand side of the gel, and reported in kbs. Samples in lanes 1-3 are AL-Y samples, in 4-6 are DR-Y samples, in 7-9 are AL-O samples, and in 10-12 are DR-O samples. Lane 13 contains the positive control provided by the kit. (B) The average values of the median TRF lengths are reported in the graph. AL, ad libitum; DR, dietary restricted; $Y$, Young (8-8.5 months) and O, Old (26-32.5 months). Error bars represent the +SE.

aged cells, probably as an indirect consequence of aging (Dimri et al., 1995). In the brain slices, we performed the SA- $\beta$-gal assay and quantified the $\beta$-gal signal (Fig. 6A). We analyzed $3 \mathrm{AL}-\mathrm{Y}$ samples (35 slices in total), 3 DR-Y samples (27 slices in total), 2 AL-O samples (11 slices in total), and 2 DR-O samples (18 slices in total). As expected the $\beta$-gal signal increased significantly with old age (Fig. 6B; $F(1,6)=6.76, p=0.041$ ), however, diet did not change the signal levels in young or old animals (Fig. 6B; $F(1,6)=0.09, p=0.78$ ). The AL-Y group had $3.23 \%( \pm 2.05, n=3)$ of the area positive for the SA- $\beta$-gal signal, DR-Y had $3.13 \%( \pm 2.06$, $n=3)$, AL-O had $11.37 \%( \pm 5.41, n=2)$, and DR-O had $9.78 \%( \pm 1.87, n=2)$. An increase in SA- $\beta$-gal signal with age is a well-established finding, and is consistent with previous reports (Kishi et al., 2008). On the other hand, it appears that the current DR does not affect cellular senescence as measured by beta galactosidase levels. 
A

Old

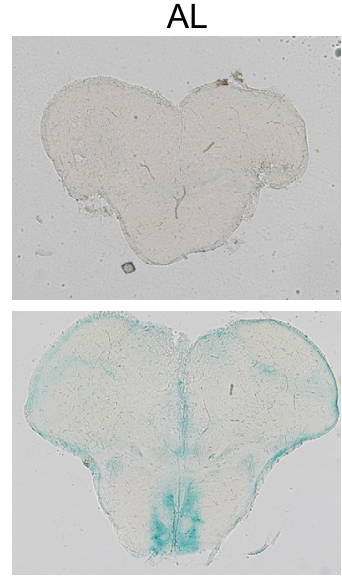

B

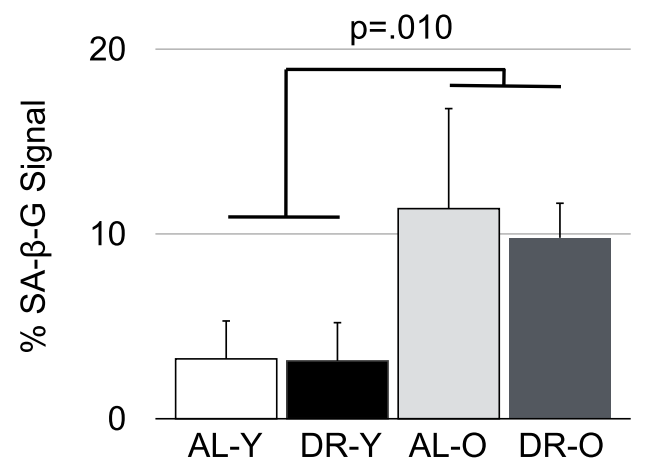

Fig. 6. The aged brain has increased levels of the cell senescence marker senescence associated $\beta$-galactosidase (SA- $\beta$-gal) that is not affected by diet. An SA- $\beta$-gal assay was performed. (A) The SA- $\beta$-gal signal (\%SA- $\beta$-gal per unit area of the section measured, $y$-axis) was quantified with ImageJ and average values were plotted. For each group 6-8 slices were measured. (B) Representative pictures of the SA- $\beta$-gal assay. Dashed lines indicate the dorsal side of the brain. Scale bar $=100 \mu \mathrm{m}$. AL-Y $(n=3)$, DR-Y $(n=3)$, AL-O $(n=2)$, and DR-O $(n=2)$. AL, ad libitum; DR, dietary restriction; Y, Young (88.5 months) and $\mathrm{O}$, Old (26-32.5 months). Error bars represent the $+\mathrm{SE}$.

\section{Characterization of the actively proliferating cells}

Our data using a 10-week every-other-day DR indicated that the number of actively proliferating cells per unit does not change significantly, but there were significant changes in telomere lengths. Therefore, we decided to characterize the actively proliferating cells to determine whether the subpopulation of these proliferating cells differ in their origin. We identified the cells as either glia or neurons. Since we detected the actively proliferating cells, i.e. cells that are proliferating in the narrow window of $4 \mathrm{~h}$, it was not possible to see the expression of any neuronal markers so we decided to count the glial cells. For this, we utilized a transgenic line, gfap: GFP, which expresses GFP under the gfap promoter. We applied the same DR regimen to the young gfap: GFP fish for 10 weeks and fed the AL group in parallel. Then, we performed BrdU injections and euthanized the fish after four hours. To distinguish the glial cells from neuronal cells, we counted only the cells that have a complete overlap in GFP and BrdU signals (arrows,
Fig. 7A). We counted only the regions 4 and 5 , since the highest differences in the cell proliferation rates were observed in these regions. We found that the glial cells accounted for $12.45 \%( \pm 3.69)$ of all the actively proliferating cells in the AL group, and $9.62 \%( \pm 0.6)$ in the DR group $(t(9)=-0.74, p=0.48$; Fig. 7B). We also calculated the ratio of proliferating cells per slice, which was approximately $16.35 \%( \pm 1.99)$ for the $\mathrm{AL}$ group and $14.44 \%( \pm 1.20)$ for DR group $(t(9)=-0.74$, $p=0.48$; Fig. $7 \mathrm{C}$ ). These numbers were for the regions 4 and 5 combined. When we separated the two regions, we found that region 4 does not differ between the $\mathrm{AL}$ and DR groups $(U=7.50, \quad z=-1.37, \quad p=0.17$, $r=-0.41$ ), whereas in region 5 , the DR group numerically had a lower percentage of glial cells but this was not statistically significant $(U=14, z=-0.18$, $p=0.86, r=-0.06)$. In region 4 , the numbers were as follows; AL; $14.72 \%$ ( \pm 3.95$)$, DR; $14.08 \%( \pm 1.16)$, and for region 5, AL; 11.54 ( \pm 3.68$)$, DR; 6.91\% ( \pm 1.14$)$; Fig. 7D). As a result, we found that DR does not change the subpopulation of the proliferating cells, or favor the proliferation of either glial or neuronal cell types in young animals.

\section{DISCUSSION}

In this study we used an every-other-day feeding DR regimen for 10 weeks in zebrafish, and analyzed the actively proliferating cells in both young and old animals. Presently, DR is quite a timely and important topic and our study provides novel findings. Firstly, we used old animals as subjects. A three-year old fish likely corresponds to a human age over 60 based on current human life expectancy at birth rates (79.56 years of age for the USA, 80.02 for the $\mathrm{EU}^{\dagger}$ ). Secondly, we used a relatively prolonged regimen of DR started later in life in the zebrafish. With this ten-week dietary regimen, we have estimated that, for a human, it would be a diet of almost 4 years, starting at the age of 60 . This is important when we think of the applicability of a DR regimen to humans. The goal was to obtain an idea of the benefits of a person starting a diet at a later age in life. Thirdly, we measured the effect of DR on the actively-proliferating cells in old animals and compared the results to young subjects. Finally, we analyzed the aging parameters related to cellular senescence, such as telomere length and SA- $\beta$-gal in young and old animals after the 10-week $\mathrm{DR}$ regimen.

We successfully applied the DR regimen to young and old zebrafish. The regimen of every-other-day feeding is a practical and widely accepted method of DR that shows similar results to protocols utilizing daily caloric reductions. Our DR paradigm is the only one utilized in zebrafish that follows similar dietary regimens to those used in rodents and our DR-treated young and old animals weighed significantly less than their AL counterparts, $25 \%$ and $19 \%$, respectively. Moreover, the body lengths did not change, indicating a normal growth pattern observed in aging animals. We found that our

$\dagger$ https://www.cia.gov/library/publications/resources/the-worldfactbook/index.html. 
A
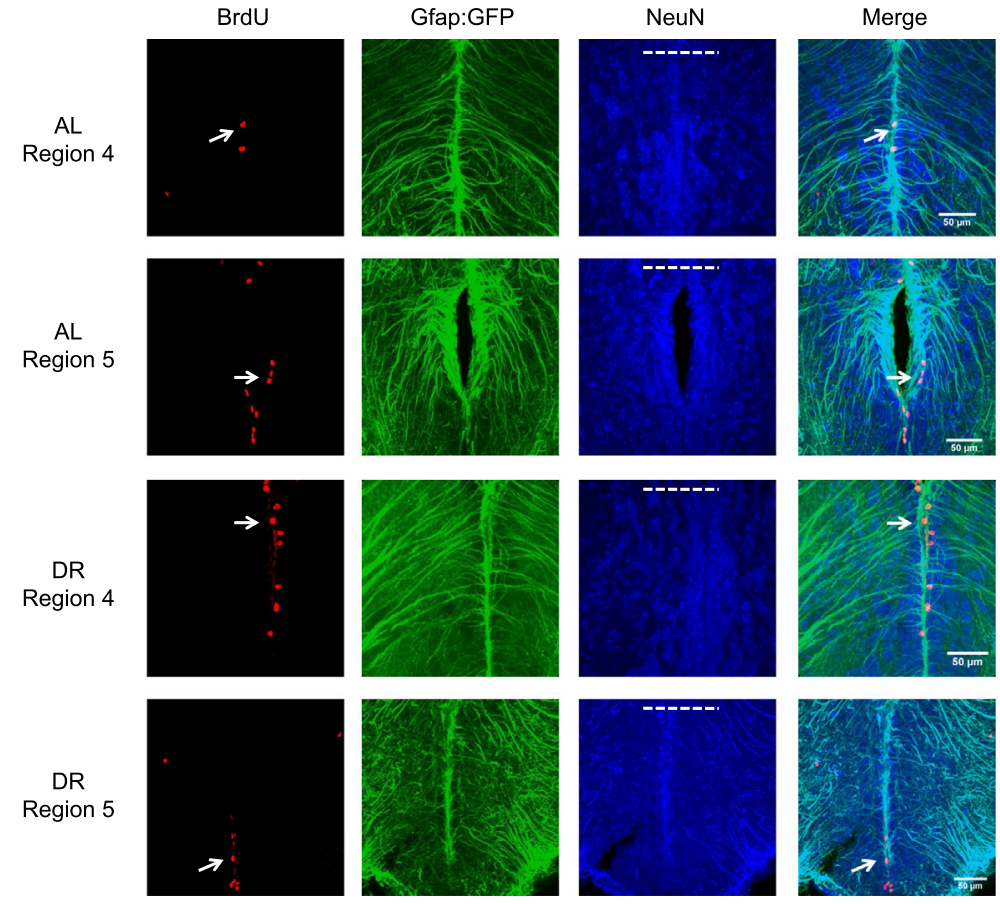

B

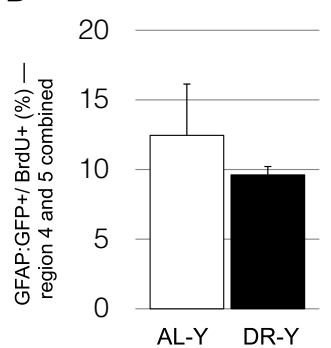

C

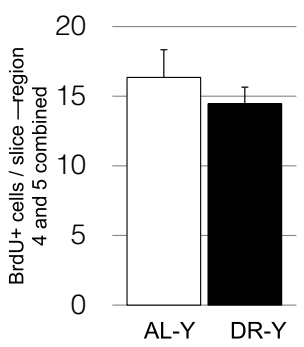

D

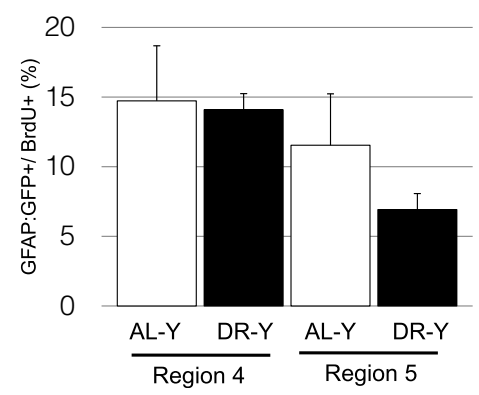

Fig. 7. Diet does not alter the subpopulation of proliferating cells. For characterization of actively proliferating cells a gfap:GFP zebrafish line was used for determining the cells of glial origin. The GFP antibody was used to detect glial cells and the NeuN antibody was used to label mature neurons. (A) Representative pictures of GFP and BrdU signals in the zebrafish telencephalon. BrdU + cells are shown in the left column, in red. In the second column from the left the gfap:GFP + cells are in green. In the third column the NeuN + cells are in blue, and the far right column shows the merged images. Glial cells, which are completely overlapping with GFP and BrdU signals, are indicated with the white arrows. Dashed lines indicate the dorsal side of the brain. (B) Among the BrdU + cells, glial cells (gfap:GFP + ) are presented as a percentage of the total number. (C) $\mathrm{BrdU}+$ cells were counted and presented as a ratio of the measured brain area in the sections. (D) Percentage of gfap:GFP + to BrdU + cells is given separately for regions 4 and 5 . Black rectangles; $A L$, white rectangles; DR. Rows 1 and 2 are $A L, 3$ and 4 are $D R ; 1$ and 3 are from region 4,2 and 4 are from region 5 . Scale bars $=50 \mu \mathrm{m}$. AL-Y $(n=5)$ and DR-Y $(n=6)$. AL, ad libitum; DR, dietary restricted; Y, Young (8-8.5 months). Error bars represent the $+\mathrm{SE}$.

DR regimen did not cause any altered stress response in the young and old fish, due to the fact that both the $A L$ and DR animals had similar cortisol level measurements. Changes in cortisol levels have been found to be correlated with stress in response to stressors such as, temperature change, osmotic shock (Yeh et al., 2013), and dominant-subordinate interaction (Pavlidis et al., 2011). Upon predator stress, cortisol levels have been reported to increase up to $16 \mathrm{ng} / \mathrm{g}$ (Barcellos et al., 2010), supporting our high levels of cortisol following the intraperitoneal injections. One explanation as to why the older fish numerically have lower cortisol levels may be due to the old fish being more resistant to injection stress, which requires further investigation. So our dietary regimen decreased weight loss significantly without disrupting the normal social hierarchy of the fish, without interfering with normal growth patterns and without inducing any stress.

We report here that DR does not affect the number of the actively proliferating cells per unit volume. We measured this as a global effect throughout telencephalon, and for individual regions of the telencephalon. This finding is in accordance with the previous publications. Seminal works coming out of the Mattson laboratory reported that DR increases neurogenesis, however, the number of cells proliferated (cells $/ \mathrm{mm}^{3}$ ) in 1 day is similar in DR and AL groups (Lee et al., 2000) or even decreased slightly in the dentate gyrus of mice (Lee et al., 2002). The increase in neurogenesis was a result of the higher rates of survival over 
4 weeks. Bondolfi et al. reported that DR did not change the number of proliferating cells in the granule cell layer $2 \mathrm{~h}$ post-BrdU injection or in the hilus, but changed it after 4 weeks post-BrdU injection and only in the hilus, not in the granule cell layer (Bondolfi et al., 2004). A recent study also reported that DR did not change the number of proliferating cells in the dentate gyrus in mice, but increased the survival rates (Kim et al., 2015). In contrast, a study using nestin-GFP mice reported an increase in the number of dividing cells in dentate gyrus of female mice (Park et al., 2013). In all of these studies, DR was started at a young adult age and continued throughout life. Our contribution to these studies is that we started our DR regimen in zebrafish at an older age, as well as at a young adult stage, and then compared the effects of the same duration of diet on both age groups.

Our results demonstrated a significant change during aging, in terms of actively proliferating cells. It has been previously reported that number of proliferating cells decreases with age in zebrafish brain (Edelmann et al., 2013). They used zebrafish of $3,6.5,7.5,10$, and 20 months of age. The decrease in the number of new neurons per olfactory bulb section was pronounced from 3 to 6.5 months, and then continued to decrease throughout life although it was at a lower rate. In the same study, by using a gfap:GFP zebrafish line, radial glia cells were found to be maintained in old age. Bondolfi et al. reported a significant decrease in total number of BrdU-positive cells in the dentate gyrus in aging mice from 2 months to 12 months of age, and the decrease continues to 24 months of age (Bondolfi et al., 2004). This finding is also in accordance with our results. Thus, our data on cell proliferation rates in young and old zebrafish are consistent with those reported in the literature.

Telomeres are the biological clocks that shorten as the organism ages. This is valid for a wide array of organisms including humans (Wright and Shay, 2005), non-human primates (Steinert et al., 2002), mouse (Rudolph et al., 1999), and zebrafish (Anchelin et al., 2011). Zebrafish are especially interesting because they have detectable telomerase expression even in the adult tissues (Kishi et al., 2003). Anchelin et al. reported a decrease during old age in telomere lengths and verified that telomerase expression is maintained in old zebrafish (Anchelin et al., 2011). We found that the DR regimen had a possible negative effect by shortening the telomeres, however the telomere lengths were not lower than the levels of the old animals, suggesting a stabilizing effect as well. This idea of DR having a reduced but stabilizing effect has been reported previously with regard to synaptic proteins in the rat hippocampus (Shi et al., 2007; Adams et al., 2008; Newton et al., 2008). Interestingly, it was reported that although telomere shortening reduces the number of proliferating cells in the brain, it has a protective effect against aging; specifically telomere shortening reduces the formation of plaques (Rolyan et al., 2011). Telomere lengths are getting shortened in the young group due to DR; since these were age-matched samples there shouldn't be any differences in the number of cell divisions. However, an increase in cell division as a result of $D R$ is a possible explanation. We also found that the telomere lengths were shorter in the brain when compared to the spleen, which is similar to humans; a study conducted in humans reported a twofold higher telomere length in the spleen as compared to the brain (Dlouha et al., 2014). This finding may indicate the reason for the differential aging dynamics of different organs.

The $\beta$-galactosidase (SA- $\beta$-Gal) assay is a widely used marker for cellular senescence. In zebrafish, this assay has been performed in the veins (Donnini et al., 2013), the whole body of the adults (Kishi et al., 2003), and larvae (Kishi et al., 2009), and skins of irradiated zebrafish (Tsai et al., 2007). In 31-month old fish, the $\beta$-Gal signal was shown to be increased (Kishi et al., 2003). To our knowledge, there are no studies that performed an SA- $\beta-$ Gal assay in brain sections. In this study, we compared the $\beta$-Gal signal between young and old zebrafish brain slices, as well as between AL and DR groups. As expected, we found that the $\beta$-Gal signal was higher in old animals as compared to young animals, however, there were no differences due to diet. This finding gives further insight into the DR mechanisms, as they do not appear to include cellular senescence at least at the $\beta$-Gal level, at least utilizing the current DR. Currently experiments are ongoing examining changes in cellular stress markers as well as different lengths of DR on cellular senescence markers.

We used a transgenic zebrafish line; gfap:GFP, which expresses GFP under the gfap promoter. gfap is an intermediate filament, which is expressed mainly in the astrocytes in the brain (Yang and Wang, 2015). Astrocytes have been found to be key players in brain aging, which is reviewed thoroughly elsewhere (Salminen et al., 2011). We analyzed the ratio of gfap-positive cells among the proliferating cells after DR. We found that the ratios using the current DR did not change in the selected regions of telencephalon. Hence, we can infer that the DR does not cause a shift in the type of cell into which they are differentiating. On the other hand, since the BrdU-positive cells are the actively proliferating cells since we immediately euthanize the fish 4-h after a BrdU injection, these cells are not likely the mature astrocytes. During the development of hippocampal neurons, gfap is expressed along with BLBP, Nestin, and Sox2 (Zhao et al., 2008). These are referred to as type 1 hippocampal progenitors, and have a different morphology than the mature astrocytes (Zhao et al., 2008). Also, it has been demonstrated that gfap-positive cells can give rise to neuroblasts and neurons (Garcia et al., 2004). Thus, actively-proliferating, gfap-positive cells may be the type 1 hippocampal progenitors, and our data indicate that DR did not change the ratio of these cells in the telencephalon.

\section{CONCLUSION}

In the current study we examined the effects of a shortterm DR on cell proliferation and cellular markers of aging in young and old zebrafish brains. Our results demonstrated that a 10-week DR, which reduces body weight significantly, does not affect the number of actively proliferating cells but alters one marker of cellular aging by shortening telomere lengths. Thus, DR 
affects the brain by subtly and occasionally altering the senescence dynamics through this mechanism and whether this is beneficial or protective remains to be determined.

\section{CONFLICT OF INTEREST}

None declared.

\section{AUTHOR CONTRIBUTIONS}

AA-E designed and performed the experiments, wrote the manuscript. BE performed the immunostaining experiments, counting and telomere measurements. DOH contributed initial planning of the experiments, did the immunostaining optimizations. ETK performed cortisol measurements, and statistical analysis. MMA conceived of the study, participated in its design, helped to finalize the manuscript. All authors read and approved the final manuscript.

Acknowledgments-This work was supported by EMBO Installation Grant to Michelle Adams and The Scientific and Technological Research Council of Turkey (TUBITAK grant number 112S649). We thank the Laura Bally-Cuif lab especially Jacob von Trotha for their help with the immunostaining protocol. We thank Adams Lab members, Melek Umay Tüz and Meriç Kınalı for their help in feeding the fish, with the injection and dissections, as well as Özge Pelin Burhan for SA- $\beta$-gal quantification.

\section{REFERENCES}

Adams MM, Donohue HS, Linville MC, Iversen EA, Newton IG, Brunso-Bechtold JK (2010) Age-related synapse loss in hippocampal CA3 is not reversed by caloric restriction. Neuroscience 171:373-382.

Adams MM, Shi L, Linville MC, Forbes ME, Long AB, Bennett $C$, Newton IG, Carter CS, Sonntag WE, Riddle DR, Brunso-Bechtold JK (2008) Caloric restriction and age affect synaptic proteins in hippocampal CA3 and spatial learning ability. Exp Neurol 211:141-149.

Adams MM, Smith TD, Moga D, Gallagher M, Wang Y, Wolfe BB, Rapp PR, Morrison JH (2001) Hippocampal dependent learning ability correlates with $\mathrm{N}$-methyl-D-aspartate (NMDA) receptor levels in CA3 neurons of young and aged rats. J Comp Neurol 432:230-243.

Anchelin M, Murcia L, Alcaraz-Pérez F, García-Navarro EM, Cayuela $M L$ (2011) Behaviour of telomere and telomerase during aging and regeneration in zebrafish. PLoS ONE 6:e16955.

Arslan Ergul A, Halim DÖ, Adams MM (2013) Bromodeoxyuridine (BrdU) labeling and immunohistochemical detection in adult zebrafish brain. Protoc Exch 12:23. Available at: http:// www. nature.com/protocolexchange/protocols/2885 [Accessed March 1, 2014].

Barcellos LJG, Ritter F, Kreutz LC, Cericato L (2010) Can zebrafish Danio rerio learn about predation risk? The effect of a previous experience on the cortisol response in subsequent encounters with a predator. J Fish Biol 76:1032-1038.

Bellesi M, Pfister-Genskow M, Maret S, Keles S, Tononi G, Cirelli C (2013) Effects of sleep and wake on oligodendrocytes and their precursors. J Neurosci 33:14288-14300.

Bondolfi L, Ermini F, Long JM, Ingram DK, Jucker M (2004) Impact of age and caloric restriction on neurogenesis in the dentate gyrus of C57BL/6 mice. Neurobiol Aging 25:333-340.

Colman RJ, Anderson RM, Johnson SC, Kastman EK, Kosmatka KJ, Beasley TM, Allison DB, Cruzen C, Simmons HA, Kemnitz JW,
Weindruch R (2009) Caloric restriction delays disease onset and mortality in rhesus monkeys. Science 325:201-204.

Dimri GP, Lee X, Basile G, Acosta M, Scott G, Roskelley C, Medrano EE, Linskens M, Rubelj I, Pereira-Smith O (1995) A biomarker that identifies senescent human cells in culture and in aging skin in vivo. Proc Natl Acad Sci U S A 92:9363-9367.

Dlouha D, Maluskova J, Kralova Lesna I, Lanska V, Hubacek JA (2014) Comparison of the relative telomere length measured in leukocytes and eleven different human tissues. Physiol Res Acad Sci Bohemoslov 63(Suppl 3):S343-S350.

Donnini S, Giachetti A, Ziche M (2013) Assessing vascular senescence in zebrafish. Methods Mol Biol Clifton NJ 965:517-531.

Edelmann K, Glashauser L, Sprungala S, Hesl B, Fritschle M, Ninkovic J, Godinho L, Chapouton P (2013) Increased radial glia quiescence, decreased reactivation upon injury and unaltered neuroblast behavior underlie decreased neurogenesis in the aging zebrafish telencephalon. J Comp Neurol 521:3099-3115.

Garcia ADR, Doan NB, Imura T, Bush TG, Sofroniew MV (2004) GFAP-expressing progenitors are the principal source of constitutive neurogenesis in adult mouse forebrain. Nat Neurosci 7:1233-1241.

Gazzaley AH, Siegel SJ, Kordower JH, Mufson EJ, Morrison JH (1996) Circuit-specific alterations of N-methyl-D-aspartate receptor subunit 1 in the dentate gyrus of aged monkeys. Proc Natl Acad Sci USA 93:3121-3125.

Gould E, Beylin A, Tanapat P, Reeves A, Shors TJ (1999) Learning enhances adult neurogenesis in the hippocampal formation. Nat Neurosci 2:260-265.

Howe K et al (2013) The zebrafish reference genome sequence and its relationship to the human genome. Nature 496:498-503.

Kempermann G, Kuhn HG, Gage FH (1998) Experience-induced neurogenesis in the senescent dentate gyrus. J Neurosci 18:3206-3212.

Kim Y, Kim S, Kim C, Sato T, Kojima M, Park S (2015) Ghrelin is required for dietary restriction-induced enhancement of hippocampal neurogenesis: lessons from ghrelin knockout mice. Endocr J 62:269-275.

Kishi S, Bayliss PE, Uchiyama J, Koshimizu E, Qi J, Nanjappa P, Imamura S, Islam A, Neuberg D, Amsterdam A, Roberts TM (2008) The identification of zebrafish mutants showing alterations in senescence-associated biomarkers. PLoS Genet 4:e1000152.

Kishi S, Slack BE, Uchiyama J, Zhdanova IV (2009) Zebrafish as a genetic model in biological and behavioral gerontology: where development meets aging in vertebrates - a mini-review. Gerontology 55:430-441.

Kishi S, Uchiyama J, Baughman AM, Goto T, Lin MC, Tsai SB (2003) The zebrafish as a vertebrate model of functional aging and very gradual senescence. Exp Gerontol 38:777-786.

Kitamura T, Mishina M, Sugiyama H (2006) Dietary restriction increases hippocampal neurogenesis by molecular mechanisms independent of NMDA receptors. Neurosci Lett 393:94-96.

Kizil C, Kaslin J, Kroehne V, Brand M (2012) Adult neurogenesis and brain regeneration in zebrafish. Dev Neurobiol 72:429-461.

Lee J, Duan W, Long JM, Ingram DK, Mattson MP (2000) Dietary restriction increases the number of newly generated neural cells, and induces BDNF expression, in the dentate gyrus of rats. $\mathrm{J} \mathrm{Mol}$ Neurosci MN 15:99-108.

Lee J, Seroogy KB, Mattson MP (2002) Dietary restriction enhances neurotrophin expression and neurogenesis in the hippocampus of adult mice. J Neurochem 80:539-547.

Lin S-J, Kaeberlein M, Andalis AA, Sturtz LA, Defossez P-A, Culotta VC, Fink GR, Guarente L (2002) Calorie restriction extends Saccharomyces cerevisiae lifespan by increasing respiration. Nature 418:344-348.

Luo J, Daniels SB, Lennington JB, Notti RQ, Conover JC (2006) The aging neurogenic subventricular zone. Aging Cell 5:139-152.

Newton IG, Forbes ME, Linville MC, Pang H, Tucker EW, Riddle DR, Brunso-Bechtold JK (2008) Effects of aging and caloric restriction on dentate gyrus synapses and glutamate receptor subunits. Neurobiol Aging 29:1308-1318. 
O'Callaghan NJ, Fenech M (2011) A quantitative PCR method for measuring absolute telomere length. Biol Proced Online 13:3.

Park HR, Lee J (2011) Neurogenic contributions made by dietary regulation to hippocampal neurogenesis. Ann N Y Acad Sci 1229:23-28.

Park J-H, Glass Z, Sayed K, Michurina TV, Lazutkin A, Mineyeva O, Velmeshev D, Ward WF, Richardson A, Enikolopov G (2013) Calorie restriction alleviates the age-related decrease in neural progenitor cell division in the aging brain. Eur $\mathrm{J}$ Neurosci 37:1987-1993.

Pavlidis M, Sundvik M, Chen Y-C, Panula P (2011) Adaptive changes in zebrafish brain in dominant-subordinate behavioral context. Behav Brain Res 225:529-537.

Rapp PR, Gallagher M (1996) Preserved neuron number in the hippocampus of aged rats with spatial learning deficits. Proc Natl Acad Sci U S A 93:9926-9930.

Rolyan H, Scheffold A, Heinrich A, Begus-Nahrmann Y, Langkopf BH, Hölter SM, Vogt-Weisenhorn DM, Liss B, Wurst W, Lie DC, Thal DR, Biber K, Rudolph KL (2011) Telomere shortening reduces Alzheimer's disease amyloid pathology in mice. Brain $\mathrm{J}$ Neurol 134:2044-2056.

Roth GS, Ingram DK, Lane MA (2001) Caloric restriction in primates and relevance to humans. Ann N Y Acad Sci 928:305-315.

Rudolph KL, Chang S, Lee H-W, Blasco M, Gottlieb GJ, Greider C, DePinho RA (1999) Longevity, stress response, and cancer in aging telomerase-deficient mice. Cell 96:701-712.

Salminen A, Ojala J, Kaarniranta K, Haapasalo A, Hiltunen M, Soininen H (2011) Astrocytes in the aging brain express characteristics of senescence-associated secretory phenotype. Eur J Neurosci 34:3-11.

Schmidt R, Strähle U, Scholpp S (2013) Neurogenesis in zebrafish from embryo to adult. Neural Develop 8:3.

Shi L, Adams MM, Linville MC, Newton IG, Forbes ME, Long A, Riddle DR, Brunso-Bechtold JK (2007) Caloric restriction eliminates the aging-related declines of NMDA and AMPA receptor subunits in the rat hippocampus and induces homeostasis. Exp Neurol 206:70-79.
Smith TD, Adams MM, Gallagher M, Morrison JH, Rapp PR (2000) Circuit-specific alterations in hippocampal synaptophysin immuno- reactivity predict spatial learning impairment in aged rats. J Neurosci 20:6587-6593.

Spalding KL, Bergmann O, Alkass K, Bernard S, Salehpour M, Huttner HB, Boström E, Westerlund I, Vial C, Buchholz BA, Possnert G, Mash DC, Druid H, Frisén J (2013) Dynamics of hippocampal neurogenesis in adult humans. Cell 153:1219-1227.

Steinert S, White DM, Zou Y, Shay JW, Wright WE (2002) Telomere biology and cellular aging in nonhuman primate cells. Exp Cell Res 272:146-152.

Terai M, Izumiyama-Shimomura N, Aida J, Ishikawa N, Sawabe M, Arai T, Fujiwara M, Ishii A, Nakamura K, Takubo K (2013) Association of telomere shortening in myocardium with heart weight gain and cause of death. Sci Rep 3:2401.

Tsai SB, Tucci V, Uchiyama J, Fabian NJ, Lin MC, Bayliss PE, Neuberg DS, Zhdanova IV, Kishi S (2007) Differential effects of genotoxic stress on both concurrent body growth and gradual senescence in the adult zebrafish. Aging Cell 6:209-224.

van Praag H (2009) Exercise and the brain: something to chew on. Trends Neurosci 32:283-290.

van Praag H, Kempermann G, Gage FH (1999) Running increases cell proliferation and neurogenesis in the adult mouse dentate gyrus. Nat Neurosci 2:266-270.

Wright WE, Shay JW (2005) Telomere biology in aging and cancer. J Am Geriatr Soc 53:S292-S294.

Wullimann MF, Rupp B, Reichert H (1996) Neuroanatomy of the zebrafish brain : a topological atlas. Basel; Boston: Birkhäuser Verlag.

Yang Z, Wang KKW (2015) Glial fibrillary acidic protein: from intermediate filament assembly and gliosis to neurobiomarker. Trends Neurosci 38:364-374.

Yeh C-M, Glöck M, Ryu S (2013) An optimized whole-body cortisol quantification method for assessing stress levels in larval zebrafish Kalueff AV, ed. PLoS ONE 8:e79406.

Zhao C, Deng W, Gage FH (2008) Mechanisms and functional implications of adult neurogenesis. Cell 132:645-660. 